\title{
STUDY OF CARDIAC STATUS IN END STAGE CHRONIC LIVER DISEASE AND ITS CORRELATION WITH MELD
}

Tribhuwan Nath Dubey¹, Ramiz Panjwani²

1 Professor and HOD, Department of Medicine, Gandhi Medical College, Bhopal.

${ }^{2}$ Post Graduate Resident, Department of Medicine, Gandhi Medical College, Bhopal.

ABSTRACT
BACKGROUND
Liver Cirrhosis is a major problem in India caused by high prevalence of alcoholism amongst the population. Cirrhotic
cardiomyopathy is used to describe features indicative of abnormal heart structure and function in patients with cirrhosis.

\section{AIMS}

Study is to assess cardiac changes in chronic liver disease patients and its correlation with MELD score.

\section{METHODOLOGY}

This study was carried out on 60 patients who were admitted in Hamidia Hospital, GMC, Bhopal, from December 2014 to December 2015. All patients were subjected to physical examination, laboratory investigations, echocardiography and twelve lead ECG. Severity of cirrhosis was evaluated by MELD score.

\section{STATISTICAL ANALYSIS}

Data were analysed using SPSS 20. P value $<0.05$ was considered as statistically significant.

\section{RESULTS}

$31.66 \%$ of patients had increased left atrial diameter, $1.7 \%$ patients had increased right ventricular diameter, $23.3 \%$ patients had increased interventricular septal thickness, $18.33 \%$ patients had increase in diastolic left ventricular diameter, 33.3\% patients had increase in left ventricular posterior wall diameter, $28.3 \%$ patients had increased pulmonary artery systolic pressure, $5 \%$ patient had ejection fraction less than 55\%, incidence of diastolic dysfunction was $50 \%$ and $41.7 \%$ patients had QTc prolongation. In our study, all the cardiac parameters correlated significantly with MELD score.

\section{CONCLUSION}

In the present study liver cirrhosis is associated with significant cardiac changes, diastolic dysfunction and QTc prolongation and all these changes significantly correlated with severity.

\section{KEYWORDS}

Cirrhosis, Cirrhotic Cardiomyopathy, MELD Scores, Echocardiography, Diastolic Dysfunction, QTc Prolongation.

HOW TO CITE THIS ARTICLE: Dubey TN, Panjwani R. Study of cardiac status in end stage chronic liver disease and its correlation with MELD. J. Evolution Med. Dent. Sci. 2016;5(47):3026-3029, DOI: 10.14260/jemds/2016/704

\section{INTRODUCTION}

Cirrhosis is a condition that is defined histopathologically and has a variety of clinical manifestations and complications, some of which can be life-threatening.(1) Cirrhosis is associated with wide range of cardiovascular abnormalities.(2) Patients of liver cirrhosis have hyperdynamic circulation manifested as high cardiac output, decreased systemic vascular resistance and widespread arterial vasodilatation. ${ }^{(3)}$

Cirrhotic cardiomyopathy is the term used to describe a collection of characters expressive of abnormal heart structure and function. $(4)$

Financial or Other, Competing Interest: None.

Submission 25-04-2016, Peer Review 20-05-2016,

Acceptance 26-05-2016, Published 13-06-2016.

Corresponding Author:

Dr. Ramiz Panjwani,

Hostel F-Block, Room No. 31,

Gandhi Medical College,

Bhopal-462001,

Madhya Pradesh

E-mail: ramiz186@gmail.com

DOI: $10.14260 /$ jemds/2016/704
Cirrhotic Cardiomyopathy is defined by following Clinical Criteria

1. Baseline increased cardiac output, but blunted ventricular response to stimuli.

2. Systolic and diastolic dysfunction.

3. Absence of overt left ventricular failure at rest.

4. Electrophysiological abnormalities including prolonged QT interval.(5)

Structural and functional cardiac abnormalities have been reported in patients with cirrhosis, irrespective of its aetiology including dilatation of both heart chambers. In particular, cirrhosis is associated with increase in left atrium and right atrium sizes and in right ventricle diastolic diameter.(6)

The liver allocation system for Orthotopic Liver Transplantation (OLT) changed from the classical Child-Pugh system to MELD score in most of the countries of the world after 2002, because direct evidence demonstrated that mortality rates of patients waiting for liver transplant were lower with implementation of MELD scoring system.(7)

The Model for End Stage Liver Disease (MELD) score provides robust estimates of mortality. It is based upon a 
logarithmic equation that represents a sum of serum values of bilirubin, International Normalized Ratio (INR) and creatinine.(8) These parameters measure liver dysfunction and correlated with severity of liver disease.

\section{MATERIAL AND METHODS}

60 diagnosed case of cirrhotic (39 male and 21 female) patients, aged between 18 years to 60 years, confirmed by ultrasonography and other biochemical parameters are selected from Medical Ward in the period of December 2014 to December 2015. Informed consent was taken from all the study participants. Approval was taken from the Ethics Committee of our Institution.

The baseline demographic and clinical features of patients are shown in Table 1.

Echocardiography was performed using quantitative Mmode and two dimensional transthoracic echocardiography and colour flow Doppler examination by same cardiologist in Department of Cardiology, Hamidia Hospital, Bhopal. Equipment used was AACUSON ultrasound and echocardiographic system advanced model equipped with Doppler tissue imaging. Each two dimensional study consisted of parasternal long axis and short axis, and apical two and four chamber views. The conventions of American Society of Echocardiography were followed in obtaining dimensions.(9) Measurement of the following parameters were performed - left atrial and right ventricular diameters, left ventricular systolic and diastolic diameters, interventricular septum, left ventricular posterior wall, ejection fraction and estimated pulmonary artery pressure. The E/A ratio (Early maximal ventricular filling velocity/Atrial maximal filling velocity) was used as an index of diastolic dysfunction and was considered abnormal when $\mathrm{E}<\mathrm{A}$.

Previous data showed that the MELD score is an accurate predictor of survival in cirrhotic patients on the waiting list for OLT and has been used worldwide as a reliable parameter for liver allocation policies, because it correlates with the decline of the liver function. Variables are Bilirubin, INR and Creatinine, are used to calculate a score range from 6 to 40 . The score is calculated according to the formula MELD $=9.57$ $\mathrm{x}$ Loge (creatinine $\mathrm{mg} / \mathrm{dL}$ ) $+3.78 \mathrm{x}$ Loge (bilirubin $\mathrm{mg} / \mathrm{dL}$ ) + $11.2 \times$ Loge (INR) + 6.43.(8) as reported. We stratified the resulting values as $<16$ or $>16$ points, because this is a usual cut of points for considering OLT.(10)

\section{STATISTICS}

The data were analysed using SPSS 20. Appropriate univariate and bivariate statistical analysis were carried out using the student's ' $t$ ' test for the continuous variable (Age) and two-tailed Fisher exact test or chi-square $\left(\mathrm{c}^{2}\right)$ test for categorical variables. To measure the linear dependence between two random variables, Pearson's correlation coefficient was used. All means are expressed as mean \pm standard deviation and proportion in percentage are expressed as mean \pm standard deviation. The critical level of significance of the results were considered at 0.05 level, i.e. $P$ $<0.05$ was considered significant.

\section{RESULTS}

\section{Cardiac Changes in Cirrhosis Patients}

Out of 60 chronic liver disease patients, 41 patients were having left atrial diameter in normal range (68.3\%), 18 patients were having mild increase in left atrial diameter (30\%), while only 1 patient was having moderate enlargement in left atrial diameter (1.7\%).

Out of 60 chronic liver disease patients, 59 patients were having right ventricular diameter in normal range (98.3\%), 1 patient was having mild increase in right ventricular diameter (1.7\%).

Out of 60 chronic liver disease patients, 46 patients were having interventricular dimension in normal range $(76.7 \%)$, 14 patients were having mild increase in interventricular dimension (23.3\%).

Out of 60 chronic liver disease patients, 49 patients were having diastolic left ventricular diameter in normal range (81.66\%), 11 patients were having mild increase in diastolic left ventricular diameter (18.33\%).

Out of 60 chronic liver disease patients, 40 patients were having left ventricular posterior wall diameter in normal range $(66.7 \%), 20$ patients were having mild increase in left ventricular posterior wall diameter (33.3\%).

Out of 60 chronic liver disease patients, 43 patients were having pulmonary artery systolic pressure in normal range $<30(71.7 \%), 17$ patients were having mild increase in pulmonary artery systolic pressure (28.3\%).

Out of 60 chronic liver disease patients, 57 patients were having ejection fraction $>55 \%(95 \%), 03$ patients were having ejection fraction $<55 \%(5 \%)$.

\section{Correlation with MELD Score - Table 2}

Left atrial diameter, right ventricular diameter, interventricular septum thickness, left ventricle end diastolic diameter, left ventricle posterior wall diameter, left ventricular systolic diameter, left ventricle ejection fraction, pulmonary artery systolic pressure significantly correlated with the MELD score (Table 2). Patients with more severe liver disease (MELD >16) were found to have higher cardiac parameters when compared with patients who had MELD $<16$; $50 \%$ of patients, 30 out of 60 were found to have diastolic dysfunction which correlate with the age, while it did not correlate with the sex, MELD score and aetiology of cirrhosis; $41.7 \%$ of patients, 25 out of 60 were found to have QTc prolongation which correlated with the MELD score, while it did not correlate with the age, sex and aetiology of cirrhosis.

\begin{tabular}{|c|c|}
\hline Variables & Data \\
\hline AGE (years) & $44.98 \pm 12.07$ \\
\hline Gender male/female, n (\%) & $39(65) / 21(35)$ \\
\hline BMI (kg/m $\left.{ }^{2}\right)$ & $21.87 \pm 4.67$ \\
\hline Aetiology- & $20(33.33)$ \\
Alcoholic, n (\%) & $15(25)$ \\
Viral, n (\%) & $8(13.33)$ \\
Both, n (\%) & $17(28.3)$ \\
Cryptogenic, n (\%) & $0(0)$ \\
Child-Pugh & $22(36.7)$ \\
A, n (\%) & $38(63.7)$ \\
\hline B, n (\%) & $20.47 \pm 8.92$ \\
C, n (\%) & $3.76 \pm 6.04$ \\
\hline MELD & $112.58 \pm 48.84$ \\
\hline Bilirubin (mg/dL) & $1.95 \pm 1.85$ \\
\hline Random blood sugar (mg/dL)
\end{tabular}




\begin{tabular}{|c|c|}
\hline Albumin (mg/dL) & $2.32 \pm 0.52$ \\
\hline PT/INR & $1.85 \pm .67$ \\
\hline Serum Sodium (mEq/L) & $135.35 \pm 6.40$ \\
\hline \multicolumn{2}{|c|}{ Table 1 } \\
\hline
\end{tabular}

Plus-minus values are mean \pm standard deviation. BMIBody Mass Index. MELD- Model for End Stage Liver Disease. PT - Prothrombin. INR - International Normalized Ratio.

The baseline demographic and clinical features of patients.

\begin{tabular}{|c|c|c|c|c|c|c|}
\hline Parameters & MELD & $\mathbf{N}$ & Mean & $\begin{array}{c}\text { Std. } \\
\text { Deviation }\end{array}$ & $t$ & $\begin{array}{c}\text { Sig. } \\
\text { (2-Tailed) }\end{array}$ \\
\hline \multirow{2}{*}{ LA } & $<16$ & 22 & 32.55 & 1.335 & \multirow{2}{*}{-11.168} & \multirow{2}{*}{.0001} \\
\hline & $>16$ & 38 & 40.82 & 3.311 & & \\
\hline \multirow{2}{*}{ RVD } & $<16$ & 22 & 17.36 & 3.360 & \multirow{2}{*}{-3.828} & \multirow{2}{*}{.0001} \\
\hline & $>16$ & 38 & 20.37 & 2.655 & & \\
\hline \multirow{2}{*}{ IVSD } & $<16$ & 22 & 7.82 & 0.395 & \multirow{2}{*}{-7.960} & \multirow{2}{*}{.0001} \\
\hline & $>16$ & 38 & 9.87 & 1.166 & & \\
\hline \multirow{2}{*}{ LVED } & $<16$ & 22 & 42.00 & 1.852 & \multirow{2}{*}{-6.894} & \multirow{2}{*}{.0001} \\
\hline & $>16$ & 38 & 49.18 & 4.666 & & \\
\hline \multirow{2}{*}{ LVPWD } & $<16$ & 22 & 7.86 & 0.560 & \multirow{2}{*}{-9.089} & \multirow{2}{*}{.0001} \\
\hline & $>16$ & 38 & 9.61 & 0.790 & & \\
\hline \multirow{2}{*}{ LVES } & $<16$ & 22 & 26.77 & 1.901 & \multirow{2}{*}{-4.911} & \multirow{2}{*}{.0001} \\
\hline & $>16$ & 38 & 30.71 & 3.463 & & \\
\hline \multirow{2}{*}{ LVEF } & $<16$ & 22 & 67.95 & 4.541 & \multirow{2}{*}{2.430} & \multirow{2}{*}{.018} \\
\hline & $>16$ & 38 & 64.24 & 6.279 & & \\
\hline \multirow{2}{*}{ PASP } & $<16$ & 22 & 21.59 & 1.368 & \multirow{2}{*}{-6.482} & \multirow{2}{*}{.0001} \\
\hline & $>16$ & 38 & 31.68 & 7.204 & & \\
\hline \multirow{2}{*}{$\begin{array}{c}\text { Diastolic } \\
\text { Dysfunction }\end{array}$} & $<16$ & 11 & & & & \multirow{2}{*}{$>0.05$} \\
\hline & $>16$ & 19 & & & & \\
\hline \multirow{2}{*}{$\begin{array}{c}\text { QTc } \\
\text { Prolongation }\end{array}$} & $<16$ & 1 & & & \multirow{2}{*}{-8.160} & \multirow{2}{*}{.0001} \\
\hline & $>16$ & 24 & & & & \\
\hline
\end{tabular}

Correlation between echocardiographic parameters and MELD score.

\section{DISCUSSION}

Study shows that echocardiographic abnormalities seen in patients with liver cirrhosis correlate directly with severity of disease assessed by MELD score. Diastolic dysfunction was seen in $50 \%$ of the patients, but no correlation was found with the severity of disease (MELD score). In ECG, QTc prolongation was seen in $41.7 \%$ of the patients; these data are consistent with previous reports.(11) and correlation was found with severity of disease (MELD score).

The demonstrable morphological and functional heart changes in patients with cirrhosis have been grouped under the term cirrhotic cardiomyopathy.(12) CCM often has an insidious course in patients with cirrhosis and no specific morphological change or mild ventricular enlargement may be present.(13) The pathological changes is due to biochemical and mechanical events that leads to gross changes in the heart, characterized by changes in geometry, mass, function and wall stress. Wall stress produces dilatation by the stimulation of a number of neuro-hormonal pathways, resulting in heart failure.(14)

Cardiac remodelling observed in patients with cirrhosis may be related to the presence of the hyperdynamic circulation which is hallmark of cirrhosis, in accordance to forward flow theory and peripheral vasodilatation hypothesis.(15)

In an echocardiography study of 24 patients with alcoholic cirrhosis, the enlarged left ventricular diameters was found both at end diastole and end systole. Increased cardiac output occurred in conjunction with an enlarged ventricle throughout the cardiac cycle, i.e. the increase in left ventricular end systolic diameters seems not to be related to the diminished afterload, but determined by increase in vascular volume.(16)

Finding in accordance with the hypothesis favouring central hypovolemia and decreased effective arterial blood volume in cirrhosis have shown that a higher portal pressure and higher hepatic blood flow independently determine a higher cardiac output. (17)

Previous publication have found left atrial, right atrial and right ventricular diastolic diameters to be significantly greater than controls, but parameters concerning left ventricular systolic dimension, septal wall and posterior wall thickness did not show significant differences.(6) Another finding was the higher pulmonary arterial pressure mechanism of increased PAP is not fully understood, but previous studies suggested that microthrombi can migrate to pulmonary vascular bed along porto-systemic shunt and can increase in vascular resistance.(18)

Right ventricle diastolic dysfunction may be due to the decrease in cardiac preload, increase in the afterload or right ventricular relaxation. Left Ventricle Diastolic Dysfunction is often linked to stiff and noncompliant ventricles which cannot accommodate the venous return to the heart in early and middle-late diastole.(19) Decreases in cardiomyocytes metabolism have been recently proposed in order to explain diastolic dysfunction.(20) Diastolic dysfunction which is considered major criteria for cirrhotic cardiomyopathy.(21) is common among patients of liver disease. In our study and previous reports. $(22,23)$, it did not correlate with severity of liver disease.

Electrophysiological abnormalities including prolonged repolarization time and impaired excitation-contraction coupling have been found in cirrhotic patients.(24) Prolonged QTc can be associated with increased risk of ventricular arrhythmias.(23) Exact mechanism is still unclear. Some studies claimed that severity of cirrhosis (MELD score) has some relations with QTc prolongation.(24) QTc prolongation and cirrhosis suggesting QTc interval as the best ECG finding in CCM.

MELD score is the reliable parameter to assess the deterioration of liver function, because it correlates with mortality.(25) In this regard, the current study demonstrates that MELD scoring system also correlates with heart changes observed in echocardiographic studies.

\section{CONCLUSION}

Liver cirrhosis is associated with echocardiographic and electrocardiographic changes in a form of dilated right cardiac chambers, diastolic dysfunction and pulmonary hypertension and QTc prolongation. Echocardiography should be part of screening of patients, because patients are at high risk of mortality and morbidity. According to relation between Child-Pugh score and MELD, we recommended echocardiography evaluation in all child B and C cirrhotic patients.

\section{REFERENCES}

1. Anthony S, Fausi H. Harrison's principles of internal medicine. Clifford lane $19^{\text {th }}$ edition, chapter 365, 2058. 
2. Celebi H, Donder E, Celikar H. Renal blood flow detection with Doppler ultrasonography in patients with hepatic cirrhosis. Arch Intern Med 1997;157(5):564-6.

3. Wang Y, Liu LP, Bai WY, et al. Renal haemodynamics in patient with liver cirrhosis assessed by colour ultrasonography. Journal of International Medical Research 2011;39:249-55.

4. Salari A, Shafaghi A, Ofoghi $M$, et al. Diastolic dysfunction and severity of cirrhosis in non-alcoholic cirrhotic patients. International Journal of Hepatology Article ID 892876 2013;p 6.

5. Pere Gines, Rober W Schrier. Renal failure in cirrhosis. N Engl J Med 2009;361:1279-90.

6. Valeriano V, Funaro S, Lionetti R, et al. Modification of cardiac function in cirrhotic patients with and without ascites. Am J Gastroenterol 2000;95(11):3200-5.

7. Huo TI, Lee SD, Lin HC. Selection an optimal prognostic system for liver cirrhosis: the model for end stage liver disease and beyond. Liver Int 2008;28(5):606-13.

8. Malinncho M, Kamath PS, Gordon FD, et al. A model to predict poor survival in patients undergoing transjugular intrahepatic portosystemic shunts. Hepatology 2000;31(4):864-71.

9. Douglas PS, DeCra JM, Devereux RB, et al. Echocardiographic imaging in clinical trials: American society of echocardiography standards for echocardiography core laboratories. J Am Soc Echocardiogr 2009;22(7):755-65.

10. Merion RM. When a patient is too well and when is a patient too sick for a liver transplant? Liver transpl 2004;10(2):S69-73.

11. Fu-Rong Sun, Ying Wang, Bing-Yuan Wang, et al. Relationship between model for end-stage liver disease score and left ventricular function in patients with end stage liver disease. Hepatobiliary Pancreat Dis Int 2011;10(1):50-4.

12. Moller S, Henriksen JH. Cirrhotic cardiomyopathy: a pathophysiological review of circulatory dysfunction in liver disease. Heart 2002;87(1):9-15.

13. Liu H, Gaskari SA, Lee SS. Cardiac and vascular changes in cirrhosis: pathogenic mechanisms. World J Gastroenterol 2006;12(6):837-42.
14. Cohn JN, Ferrari R, Sharpe N. Cardiac remodelling concepts and clinical implications: a consensus paper from an international forum on cardiac remodelling. J Am Coll Cardiol 2000;35(3):569-82.

15. Schrier RW, Arroyo V, Bernardi M, et al. Peripheral arterial vasodilatation hypothesis: a proposal for the initiation of renal sodium and water retention in cirrhosis. Hepatology 1988;8(5):1151-7.

16. Lewis FW, Adair O, Rector WG. Arterial vasodilatation is not the cause of increased cardiac output in cirrhosis. Gastroentrology 1992;102(3):1024-9.

17. Moller S, Hobolth L, Winkler C, et al. Determinants of the hyperdynamic circulation and central hypovolemia in cirrhosis. Gut 2011;60(9):1254-9.

18. Krowka MJ, Cortese DA. Hepatopulmonary syndrome an evolving perspective in the era of liver transplantation. Hepatology 1990;11(1):138-42.

19. Abd-El-Aziz TA, Mohamed Abdou, Fathy A, et al. Evaluation of cardiac function in patients in liver cirrhosis. Intern Med 2010;49(23):2547-52.

20. Papastergiou V, Skorda L, Lisgos P, et al. Ultrasonographic prevalence and factors predicting left ventricular diastolic dysfunction in patients with liver cirrhosis: is there a correlation between the grade of diastolic dysfunction and the grade of liver disease? Scientific World Journal 2012;2012:615057. doi:10.1100/2012/615057.

21. Zardi EM, Abbate A, Zardi DM, et al. Cirrhotic cardiomyopathy. J Am Coll Cardiol 2010;56(7):539-49.

22. Odilson Marcos Silvestre, Fernando Bascal, Dannusa de Souza Ramos, et al. Impact of severity of end-stage liver disease in cardiac structure and function. Annals of Hepatology 2013;12(1):85-91.

23. De BK, Majumdar D, Das D, et al. Cardiac dysfunction in portal hypertension among patients with cirrhotic and non-cirrhotic portal fibrosis. J Hepatol 2003;39(3):3159.

24. Ma Z, Lee SS. Cirrhotic cardiomyopathy: getting to the heart of the matter. Hepatology 1996;24(2):451-9.

25. Braverman AC, Streiner MA, Picus D, et al. High-output congestive heart failure following transjugular intrahepatic portal-systemic shunting. Chest 1995;107(5):1467-9. 